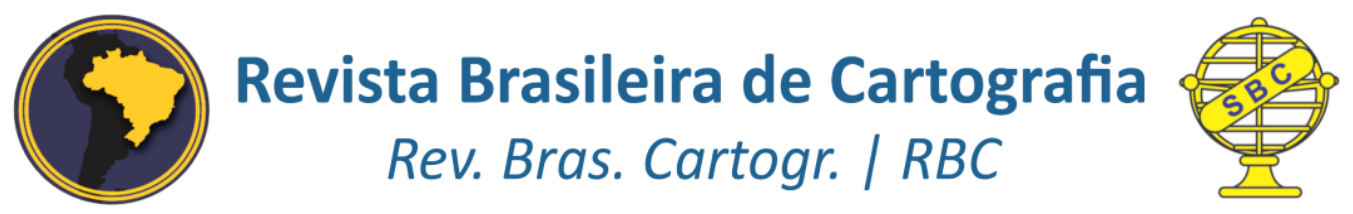

DOI: http://dx.doi.org/10.14393/rbcv71n3-48782

\title{
Desenvolvimento de Interface para Automação da Coleta de Temperatura Baseada em Redes Multiestação Aplicada ao Monitoramento de Estruturas Empregando- se Estações Totais
}

Interface Development for the Automation of Temperature Measurement Based on Multi-station Network Applied to Structure Monitoring Using Total Stations

Felipe Andrés Carvajal Rodríguez ${ }^{1}$

Luis Augusto Koenig Veiga ${ }^{2}$

Recebido em maio de 2019. Aprovado em agosto de 2019.

\begin{abstract}
RESUMO
O monitoramento geodésico de estruturas através de técnicas como irradiação tridimensional ou trilateração envolve as coletas de temperatura, pressão e umidade para cálculos referentes à correção de primeiravelocidade na medida da distância. Tradicionalmente, estas medidas são realizadas de forma pontual, junto ao equipamento de medida. Neste trabalho é apresentado o desenvolvimento de uma interface computacional para o uso integrado com a estação total em tempo real, que permite a coleta de valores de temperatura através de uma rede externa sem fio, composta por cinco sensores de temperatura, além do cálculo da correção de primeira velocidade, o qual é aplicado às distâncias medidas durante o levantamento. Para validar a aplicação do sistema proposto (software de interface mais os sensores de temperatura), os resultados de um experimento desenvolvido durante o monitoramento geodésico de uma barragem foram analisados. No processo de medição, foram obtidos diferentes valores de temperatura em cada sensor que compõe a rede, conforme a sua localização espacial na área de estudo, indicando que o uso do sistema proposto permite uma melhor e mais representativa estimativa da temperatura a ser utilizada no cálculo da correção e em tempo real. Resultados para o estudo de caso mostram variações de milímetros na correção, quando esta é comparada com o tradicional processo de mensurar os parâmetros ambientas somente na posição do instrumento de medida.
\end{abstract}

\footnotetext{
1,2 Universidade Federal do Paraná. Programa de Pós-graduação em Ciências Geodésicas, Brasil. E-
} mail: felipe.carvajalro@gmail.com,kngveiga@gmail.com. 
PALAVRAS-CHAVE: Estação-total. Monitoramento. Correção da Primeira Velocidade. Sensores de temperatura. Rede sem fio.

\begin{abstract}
The geodetic structure monitoring, using techniques such as threedimensional polar surveying or trilateration, involves the measurement of temperature, pressure and humidity for calculations referring to first velocity correction in distance measurement. Traditionally, these measures are done at the surveying instrument position. However, in this paper we present the development of a computer interface for integrated use with the total station at real time that allows the measurement of temperature values through a wireless external network, composed by five temperature sensors, and the calculation of the first velocity correction, which is applied to the distances measured during the measurement process. To validate the proposed system (interface software and temperature sensors) the results of an experiment developed during the geodetic monitoring of a dam were analyzed. In the measurement process different temperature values were obtained in each network sensor, according to its spatial location in the study area, which indicates that the use of the proposed system allows a better and more representative estimation of the temperature to be used to work out the correction, and in real time. The results for the experiment show variations of millimeters in the correction, when these are compared with the traditional process of measuring the environmental parameters only in the position of the total station.
\end{abstract}

KEYWORDS: Total Station. Monitoring. First velocity correction. Temperature sensor. Wireless network.

\title{
Introdução
}

O desenvolvimento tecnológico no campo da instrumentação geodésica tem permitido a automatização de múltiplos procedimentos, melhorando a precisão, diminuindo erros e aumentando a produtividade. Atualmente, no mercado de estações totais encontram-se equipamentos contendo servomotores e tecnologia para o reconhecimento automático de alvos (prismas), os quais permitem a realização da medição de malhas de pontos, semelhantes aos lasers scanners, com um alto grau de automação. Paralelamente aos avanços tecnológicos, os fabricantes vêm desenvolvendo softwares de trabalho padronizados e específicos para o controle das estações totais em diferentes áreas de aplicação de engenharia, como nos levantamentos topográficos, nas redes topográficas, túneis, estradas, monitoramento estrutural, entre outros 
que podem ser utilizados pelo segmento de usuários destes instrumentos (KENNIE e PETRIE ,1993).

Outra característica relevante de alguns instrumentos é a possibilidade do desenvolvimento de softwares externos ao equipamento de medida, a fim de possibilitar o controle das ações do mesmo, como, por exemplo, na realização da pontaria automática em um alvo. Através da criação de novas aplicações, orientadas de acordo com as necessidades do usuário, pode-se desenvolver diferentes rotinas de trabalho. Isto é possível graças aos chamados protocolos de comunicação, que definem regras, comandos e variáveis para a conexão de uma estação total com um computador ou aplicativo. Um exemplo é o protocolo de comunicação GeoCOM da Leica Geosystems, que permite desenvolver aplicativos para a comunicação e controle de estações totais (LEICA GEOSYSTEMS, 2009). Assim, é possível criar soluções computacionais que interajam com o instrumento de medida e que também permitam a integração com sensores externos conectados ao mesmo software.

Embora esta tecnologia facilite as operações de campo, as mensurações ainda estão sujeitas as influências dos fatores externos, como o efeito da refração e alteração na velocidade de propagação da onda eletromagnética, principal método utilizado na medição de distância, no qual devem ser considerados os parâmetros de pressão, temperatura e umidade.

A influência de cada um dos parâmetros atmosféricos na qualidade das observações geodésicas terrestres, em particular, nas distâncias obtidas pela propagação de ondas eletromagnéticas, foi analisada por Rüeger (1990) através da diferenciação da equação do índice de refração. Os resultados mostram que para uma variação unitária nos valores de temperatura, pressão e umidade, considerada pelo autor como padrão $\left(t=15^{\circ} \mathrm{C}, \mathrm{p}=1107 \mathrm{mbar}, \mathrm{h}=13\right.$. mbar), as incertezas no índice de refração são de 1 ppm, 0,3ppm e 0,04ppm, respectivamente. Ogundare (2015) realizou novas análises mediante ao método da diferenciação do índice de refração com valores de temperatura $\left(15^{\circ} \mathrm{C}\right)$, pressão (1015 mbar) e umidade (10 mbar), obtendo incertezas de 
0,97ppm, 0,28ppm e 0,038ppm, respectivamente. Em ambos casos, as mudanças na temperatura foram os mais influentes no cálculo do índice de refração.

Outra análise pode ser realizada a partir das camadas atmosféricas geradas pelos gradientes explicados por Torge (2012). Estes gradientes variam em função da altura com valores de $-0.0065^{\circ} \mathrm{C} / \mathrm{m},-0.12 \mathrm{hPa} / \mathrm{m}$ e $0.0035 \mathrm{hPa} / \mathrm{m}$ para os três fatores. Tendo em vista que os gradientes são considerados valores médios, a temperatura apresenta uma maior variação às mudanças de altura. Nesta mesma linha de pesquisa, os fenômenos de turbulência térmica associados ao gradiente da temperatura apresentados por Brunner e Fraser (1977); Angus-Leppan e Brunner (1980) e Fraser (1981), explicam o comportamento irregular deste parâmetro na definição de camadas atmosféricas, tornando-a o parâmetro que mais afeta a determinação do índice de refração.

Para Angus-Leppan (1972) e Rüeger (1990), a medição da temperatura em um extremo ou em ambos extremos da linha visada não representa a temperatura ao longo do comprimento desta, além das diferenças de altura que também geram diferença na temperatura. Para os parâmetros de pressão e umidade, os mesmos autores consideram que os valores em um extremo da linha são suficientes.

A respeito do tratamento dos parâmetros atmosféricos na determinação do índice de refração, Brunner (1984) define três áreas de pesquisa; (1) soluções instrumentais, (2) modelamento matemático e (3) desenvolvimento de novas teorias. No caso das soluções instrumentais, na atualidade, as estações totais integram microcomputadores que automatizam o cálculo do índice de refração, mediante dados atmosféricos medidos através de sensores atmosféricos externos e inseridos manualmente pelo usuário. Nesta mesma linha, a integração de sensores externos é possível devido ao desenvolvimento tecnológico na área de sensores programáveis somado à disponibilização de protocolos de comunicação para o controle de instrumentos topográficos. A integração de sensores de baixo custo foi apresentada por Carvajal (2018) 
para o controle da temperatura durante o monitoramento geodésico de estruturas com estações totais. Outro exemplo foi apresentado por Solarić, Barković e Zrinjski (2012), no qual a coleta de parâmetros atmosféricos foi automatizada e integrada no processo de calibração das estações totais na universidade de Zagreb, Croácia.

No caso do modelamento matemático, o método de linha de referência proposto por Robertson (1977) considera parâmetros atmosféricos fixos durante um intervalo de 30 minutos observado em uma linha de referência. Artese e Perrelli (2018), apresentam um modelo numérico de correção em função da integração dos parâmetros atmosféricos medidos durante o período de um ano. No caso do desenvolvimento de novas teorias, os modelos desenvolvidos em função do gradiente de temperatura fornecem resultados mais representativos e precisos do comportamento das camadas atmosféricas. Neste contexto, Brunner e Fraser (1977) apresentam o modelo turbulento de transferência (TTM) para ondas eletromagnéticas do espectro visível e microondas. Baseados na mesma teoria, Angus-Leppan e Brunner (1980) desenvolveram o modelo atmosférico de temperatura para comprimentos curtos (STREAM), que analisa, além do gradiente vertical de temperatura, diferenças entre o índice de refração teórico e calculado para observações de distâncias de até 3 quilômetros. Outro modelo baseado na teoria do gradiente vertical de temperatura é o modelo atmosférico simples de duas camadas (STAM) desenvolvido por Fraser (1981).

Neste trabalho, investigou-se uma solução instrumental para a automação da coleta de temperatura e sua integração, em tempo real, no processo de medição de distâncias durante o monitoramento geodésico de estruturas. Para tal, desenvolveu-se um software a fim de permitir o gerenciamento da estação total e de um sistema de aquisição de temperatura por meio de uma rede de comunicação sem fio, com sensores instalados em diferentes pontos de interesse. Os valores de pressão e umidade foram medidos de forma tradicional (extremo da linha em que é instalada a estação 
total), uma vez que estes apresentam comportamentos regulares nas camadas mais baixas da atmosfera (ANGUS-LEPPAN ,1972; RÜEGER, 1990).

\section{Interfaces e Protocolos}

Quando um software é desenvolvido para ser usado junto à um equipamento de medição, faz-se necessário entender o conceito de interface, a forma com que dois sistemas diferentes (computador/equipamento de medição) interagem ou ainda método de interação do sistema com o usuário. Assim três classes de interfaces podem ser definidas: interface de hardware, de software e do usuário.

A interface de hardware permite estabelecer uma conexão perfeita entre o computador e o equipamento de medição. Já a interface do usuário aborda a maneira pela qual o usuário irá interagir com o sistema desenvolvido. Decodificar dados recebidos e obter meios para enviar comandos ao equipamento correspondem à interface de software (VEIGA, 2000).

A interface do usuário deve prever a maneira como os usuários podem interagir com o sistema (instrumento/computador/software) e como os resultados dessa interação são apresentados. Devem ser levadas em conta as diferenças entre os usuários e suas necessidades específicas.

A interface de hardware refere-se à conexão física entre as unidades; envolve os tipos de cabos e conectores utilizados, bem como o método de transmissão de dados. A conexão entre dispositivos acontece através de cabos ou por comunicação sem fio, como Bluetooth ou outros sistemas de rádio. Porém, conexão física entre dois sistemas não garante que ambos possam decodificar as informações recebidas: neste ponto torna-se importante a interface do software (ou programação). A interface é o elemento responsável pela troca de informações entre os dois sistemas e de sua posterior interpretação de forma correta. Isto se dá por meio do protocolo de dados específico de cada equipamento. 
Como exemplos de protocolos padrão tem-se o NMEA 0183 (acrônimo de National Marine Electronics Association), um padrão que permite a comunicação de dados entre equipamentos eletrônicos marítimos, instrumentos de navegação e equipamentos de comunicação (NMEA 0183, 2002). No caso do GPS (Global Positioning System), o emprego do padrão NMEA é definido como padrão para diferentes fabricantes de equipamentos, o que permite o desenvolvimento de aplicativos que irão funcionar com diferentes modelos de receptores, além de facilitar o trabalho do desenvolvimento destes aplicativos. No caso de estações totais, não existe um protocolo único que permita trabalhar com modelos de diferentes fabricantes.

Um protocolo, por sua vez, é uma descrição formal dos formatos de dados, variáveis, mensagens e comandos, incluindo regras que permitem a troca de informações entre o instrumento de medição e o computador. Um exemplo de modelo de protocolo é a especificação quanto a forma de armazenamento das observações em um instrumento (o formato de dados de cada equipamento) que, no caso das estações totais, pode definir formatos padrão para cada um dos seguintes tipos de dados (VEIGA, 2000):

a) Dados de observação: são os valores medidos ou observados (como direções horizontais, ângulos verticais e distâncias inclinadas), bem como os valores estimados a partir dos mesmos;

b) Dados de controle ou operação: são dados utilizados como instruções para que o equipamento realize uma determinada tarefa, como medir a distância ou verificar a inclinação de um dos eixos;

c) Dados de configuração: através destes dados, é possível definir parâmetros internos do equipamento, como altura do instrumento, formato de medida e apresentação de distâncias e dos ângulos, etc;

d) Dados de simulação: permitem que uma tecla específica do equipamento seja emulada. Com este tipo de dado, é possível elaborar um teclado virtual que simule o teclado do instrumento, permitindo a operação diretamente na tela do computador. 
Com o surgimento das estações totais robotizadas, os dados de controle e operação podem ser subdivididos em dois conjuntos:

a) Medição: Está relacionada com a medição de uma quantidade, como direções ou distâncias;

b) Movimento: Refere-se ao desempenho dos movimentos dos instrumentos, como a inclinação da luneta ou a busca de um alvo.

Normalmente, para cada protocolo, um grupo de dados é definido e representado por uma sequência de caracteres no formato ASCII. Como exemplos de protocolos tem-se o GeoCOM (LEICA GEOSYSTEMS, 2009) e o GSI - Geo Serial Interface (LEICA GEOSYSTEMS, 1999) da Leica. Durante o desenvolvimento das aplicações, é importante verificar o protocolo específico de cada instrumento para determinar as potencialidades de cada aplicação. Neste trabalho, foi utilizado o protocolo Leica GeoCOM.

\subsection{Leica GeoCOM}

Leica GeoCOM é um protocolo de comunicação serial que permite o desenvolvimento de aplicações para o controle de estações totais Leica. O GeoCOM é baseado na chamada de procedimento remoto (RPC) desenvolvida pela SUN Microsystems. Este permite a interação entre dois terminais ponto a ponto, definindo uma comunicação síncrona na qual existem solicitações e respostas, não permitindo o envio de múltiplos requisitos ao mesmo tempo (LEICA GEOSYSTEMS, 2009)

$O$ GeoCOM define os terminais de comunicação como servidor e cliente: o servidor corresponde ao instrumento (estação total) e o cliente corresponde ao software de controle desenvolvido. O sistema é apresentado na Figura 1. 
Figura 1 - Terminais de comunicação servidor e cliente

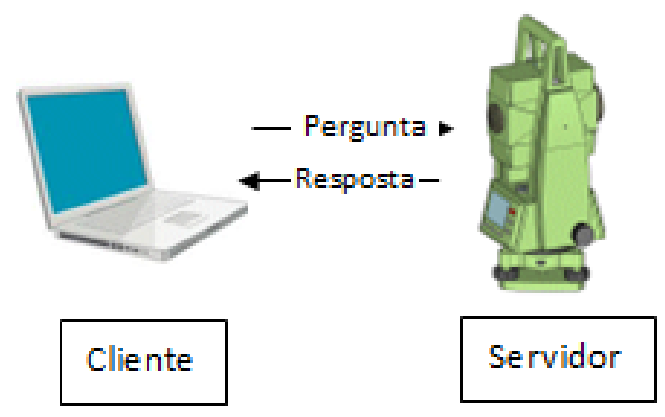

Fonte: Carvajal (2018).

O protocolo GeoCOM pode ser aplicado a várias linguagens de programação, como a VBA, C/C\# ou via protocolo ASCII. Neste último caso, a estrutura das mensagens de comunicação possui um formato ASCII linear, portanto, o cliente envia uma requisição em um formato específico e o servidor responde no mesmo formato. Na Figura 2 é apresentado o envio e recepção de códigos ASCII.

Figura 2 - Envio e recepção de códigos ASCII

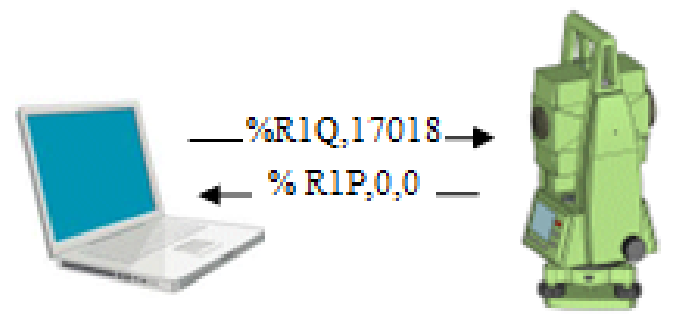

Fonte: Carvajal (2018).

Quando o cliente envia a string de texto $\% R 1 Q, 17018$, a configuração atual do medidor de distância eletrônico está sendo solicitada e a resposta é $\boldsymbol{\% R 1 P , 0 , 0}$. A parte $\mathbf{0 , 0}$ da sequência corresponde ao modo IR Std (infravermelho) do medidor eletrônico de distância. Em geral, cinco 
configurações podem ser diferenciadas para os requisitos feitos pelo cliente e suas respectivas respostas (LEICA GEOSYSTEMS, 2009):

a) Configuração: permite configurar algumas funções da estação total;

b) Verificação: permite verificar as configurações reais da estação total;

c) Inserção: permite inserir parâmetros para a configuração da estação total;

d) Obtenção: permite rever as configurações de observação atualmente empregadas;

e) Posicionamento do Telescópio: permite configurar e controlar o sistema servo-assistido, bem como o sistema de mira automática.

Maiores detalhes sobre este protocolo podem ser encontrados em LEICA GEOSYSTEMS (2009).

\section{Desenvolvimento de um software para o controle de estações totais}

O software desenvolvido para o controle de estações totais foi programado mediante aos códigos de controle do protocolo GeoCOM,

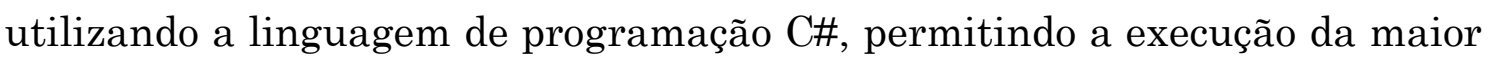
parte das funcionalidades básicas para uma estação total (orientação, levantamento de pontos, configuração de altura do prisma e instrumento, mudanças de constante de prismas, entre outras.).

A estrutura do software considera 5 funções gerais: Comunicação (Comn), Configuração (Conf), Inserção (Insr), Obtenção (Obte) e Exportação (Expt). Estas funções possuem um modo de operação bidirecional, permitindo o correto fluxo de funcionamento do processo de comunicação. Ainda, uma função foi desenvolvida para a integração de dados de temperatura por uma rede externa de sensores, denominada função temperatura (Temp). Esta, na 
estrutura proposta do software, corresponde a uma função secundária e dependente das funções gerais.

$\mathrm{Na}$ hierarquia da estrutura aplicada ao desenvolvimento do software, a função Comn ocupa a parte principal, por realizar a comunicação entre o usuário e o instrumento e, portanto, condiciona as demais funções. Os protocolos de comunicação para esta função foram escolhidos considerando códigos de controle disponíveis pelo protocolo GeoCOM da Leica, os quais permitem comunicação serial RS232 e Bluetooth. Na Figura 3, é apresentada a estrutura geral do software.

Figura 3 - Estrutura do software

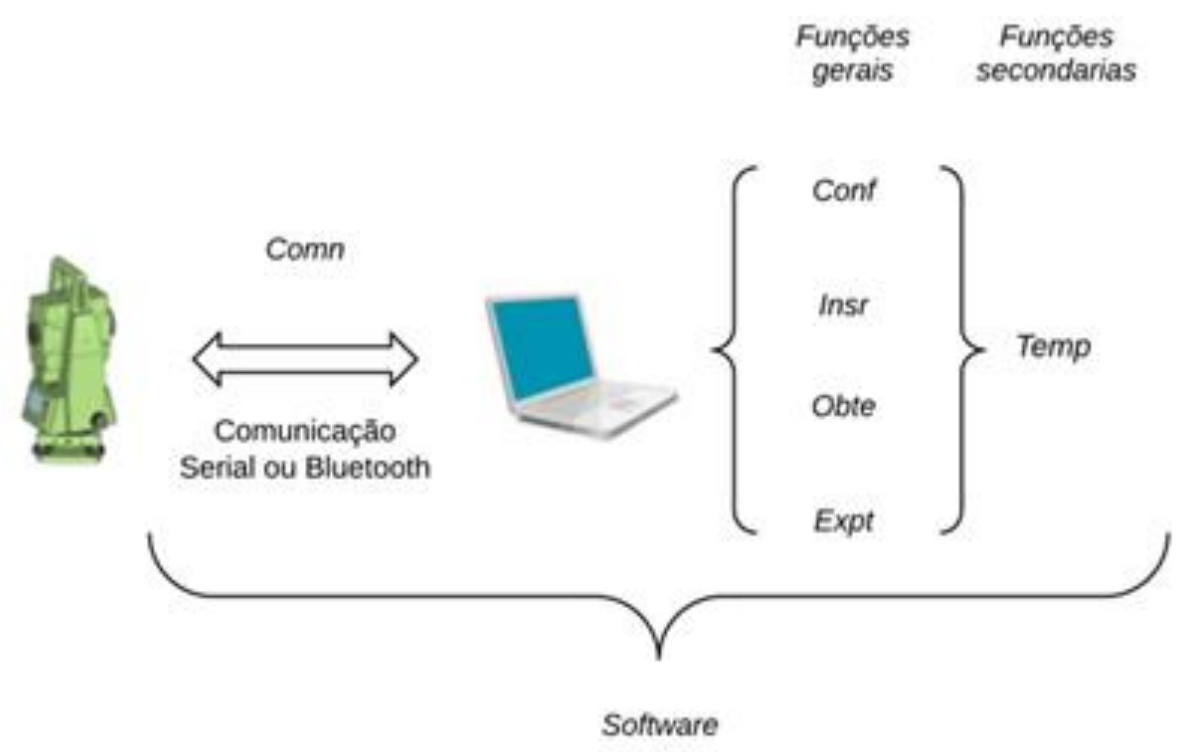

Fonte: Elaborada pelos autores.

As demais funções ficaram com o mesmo nível de hierarquia. Para o caso das funções Conf e Insr, permitem configurar (modo de medição, prisma, unidades, etc.) e inserir parâmetros (altura do prisma, altura do instrumento, etc.) respectivamente. As funções Obte e Expt têm como objetivo a 
visualização e o gerenciamento (exportação) dos dados e informações, respetivamente.

\section{Integração da Rede de Sensores de Temperatura}

Para o caso das configurações correspondentes à integração de dados atmosféricos (temperatura, pressão e umidade), o código de controle tem o seguinte formato:

$$
\% \mathrm{R} 1 \mathrm{Q}, 2028: 0, \mathrm{P}, \mathrm{T}, \mathrm{H}
$$

onde, $\mathrm{P}$ é a pressão em mbar, $\mathrm{T}$ é a temperatura em graus Celsius e H é a umidade em porcentagem. Objetivou-se a inserção do valor da temperatura a partir da observação de uma rede de monitoramento de forma automática e simultânea à realização das observações de distância.

A rede de aquisição de temperatura projetada foi composta por 5 módulos ou nodos sem fio e um módulo central. Cada módulo da rede era composto por sensores de temperatura modelo DS18B20, com uma precisão nominal de $0.5^{\circ} \mathrm{C}$, um controlador Arduino e um módulo de radiofrequência Xbee, para administrar e comunicar os valores de temperatura, respectivamente. O módulo central, por sua vez, administrou todas as informações da rede.

A rede forneceria, em tempo real, valores de temperatura de diferentes posições onde os sensores são instalados. A Figura 4, apresenta um modelo de distribuição para a estrutura da rede. 
Figura 4 - Rede de aquisição de temperatura sem fio

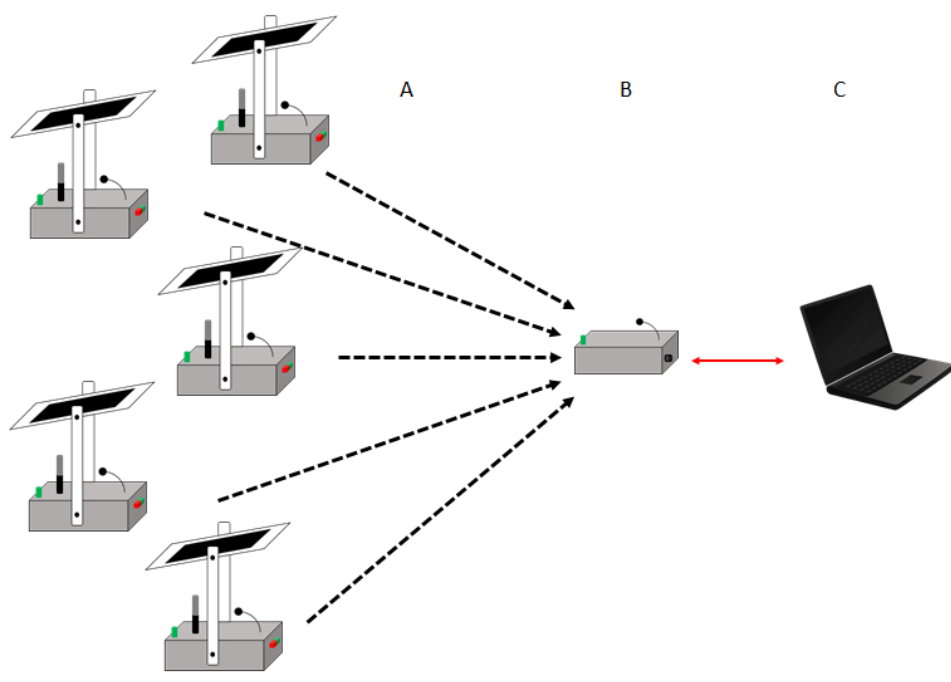

Fonte: Elaborada pelos autores.

Na Figura 4, a estrutura é dividida em três segmentos: o segmento A corresponde aos sensores que formam a rede, o segmento B sendo encarregado pelo controle da mesma, e o segmento $\mathrm{C}$ referente a manipulação, visualização e salvamento dos valores de temperatura fornecidos pelos nodos da rede. Mais informações podem ser encontradas em Carvajal (2018).

A integração da rede de sensores ao software, que permite o controle da estação total, foi realizada mediante a função secundária Temp. Esta função utiliza o código \%R1Q,2028:0,P,T,H para automatizar o processo, deixando o parâmetro de $\mathrm{T}$ em função dos sensores de temperatura. No diagrama da Figura 5, é apresentado o fluxo de trabalho. 
Figura 5 - Integração da rede de aquisição de temperatura ao fluxo de trabalho da estação total

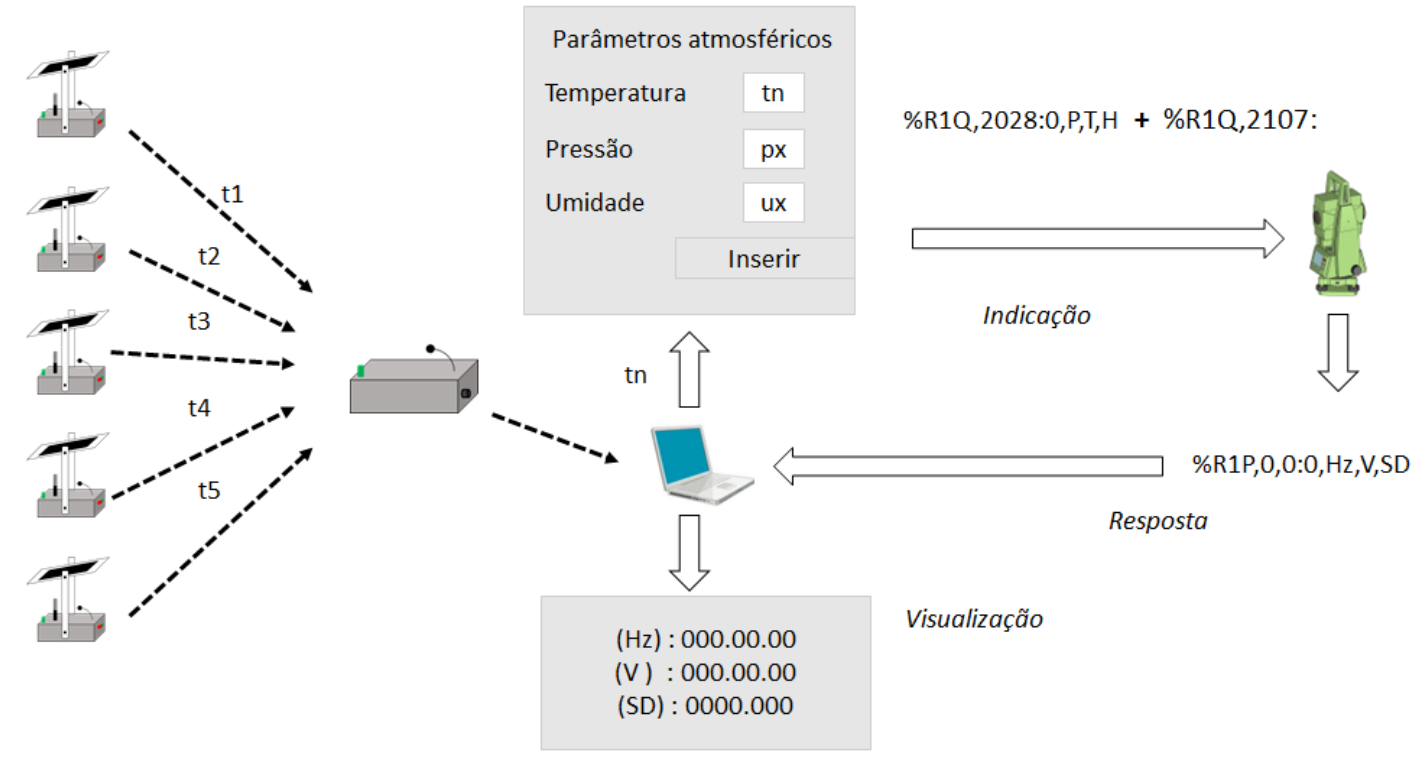

Fonte: Elaborada pelos autores.

Na Figura 5, a rede de sensores e o coordenador central da rede estão ligados ao computador que contém o software desenvolvido, permitindo a sincronização das leituras dos parâmetros atmosféricos previamente a uma medição de distância. Neste caso, os parâmetros de pressão ( $p x$ ) e umidade (ux) são inseridos de forma manual no início do conjunto de medições, enquanto $t h$ corresponde ao valor de temperatura proveniente da rede, o qual é atualizado para cada observação realizada, mediante à diferentes modelos matemáticos (media, média ponderada, valores singulares, etc.). O diagrama da Figura 6, apresenta o fluxo de trabalho desta integração. 
Figura 6 - Fluxo de trabalho final da integração

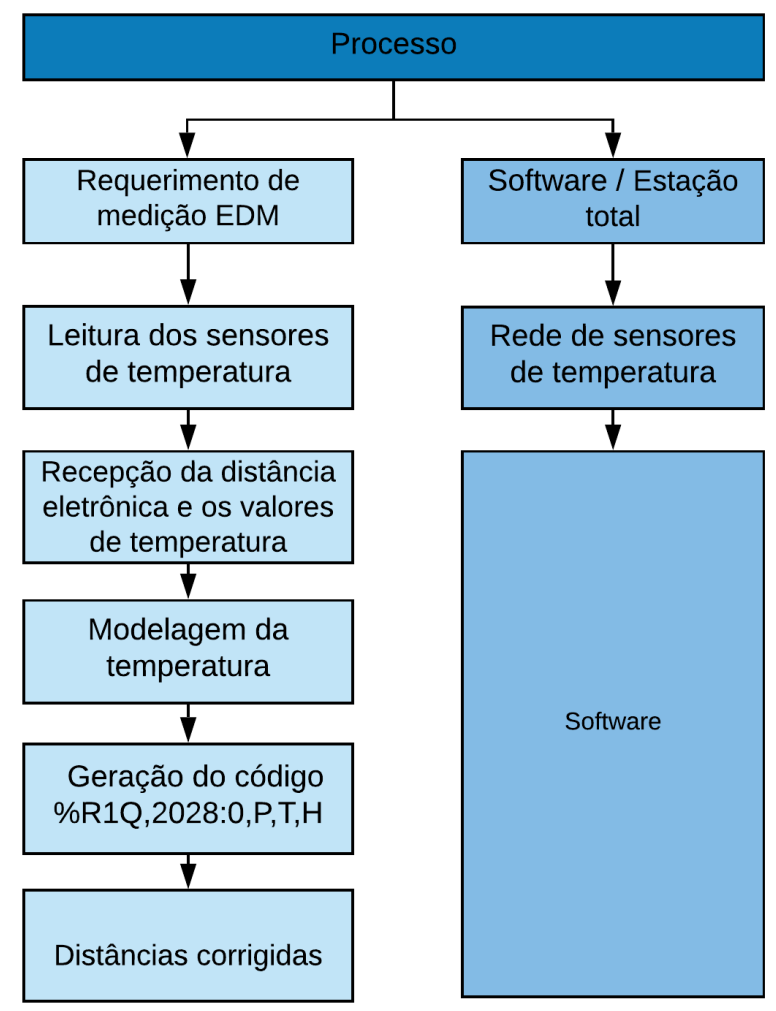

Fonte: Carvajal (2018).

Na Figura 7 é apresentada a interface do software desenvolvido, sendo indicadas as 4 regiões contendo as 5 funções. Na região A é possível configurar os parâmetros de comunicação Comn, contendo também um terminal que permite o envio e recepção de códigos de controle brutos diretamente no formato ASCII. Ainda na mesma região, existem outras abas, dentre elas a aba "Set" contendo as funções de Conf. Na região B são configuradas as coordenadas da estação e parâmetros como a altura do instrumento e do alvo. Neste caso, as funções Insr e Obte estão presentes. 
Figura 7 - Tela do software desenvolvido

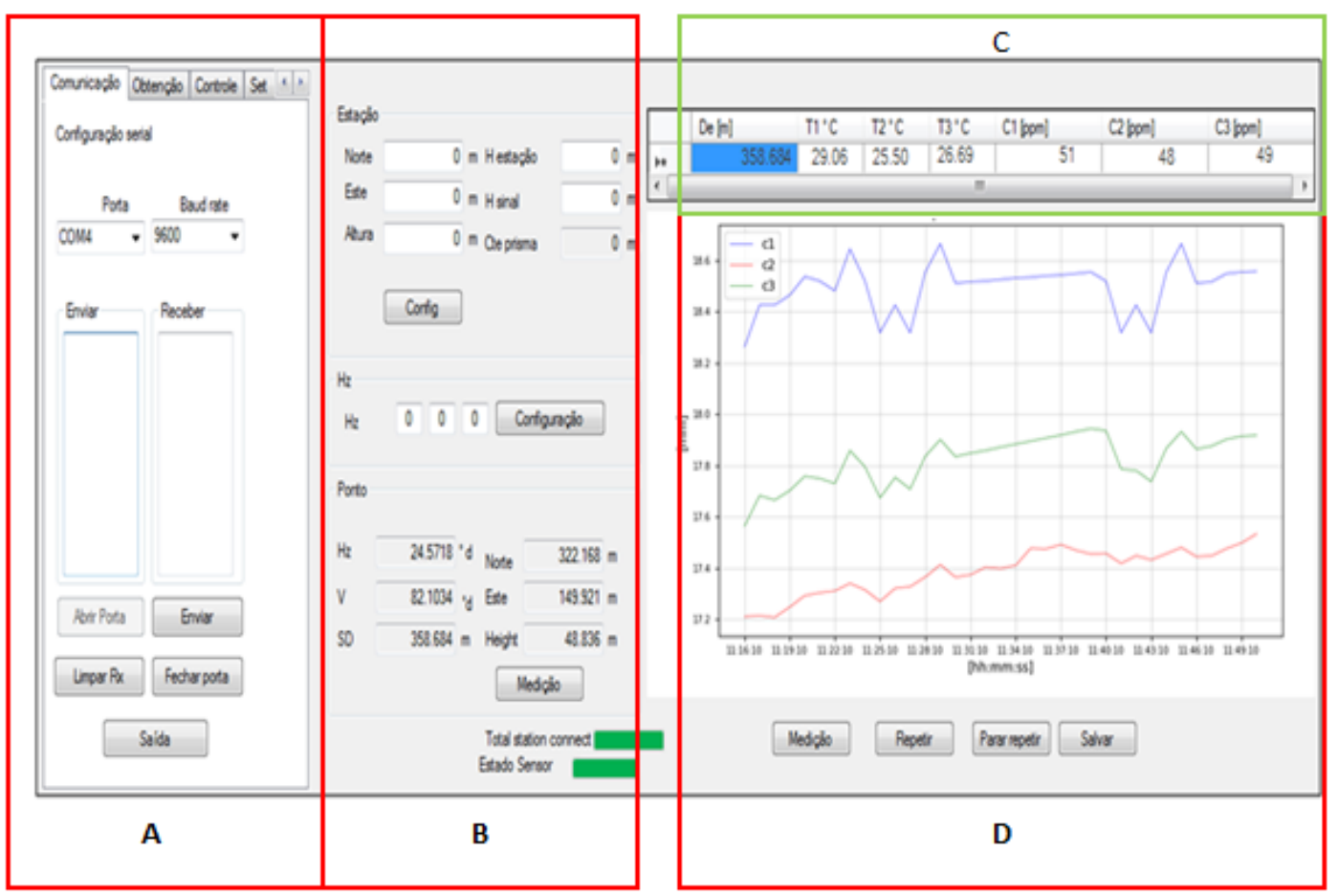

Fonte: Elaborada pelos autores.

Na região C é possível visualizar os valores da distância fornecida pela estação total, temperatura dos sensores da rede e correções atmosféricas calculadas. Já na região $\mathrm{D}$, apresenta-se um gráfico com as variações das correções atmosféricas no decorrer do tempo, além da opção "Salvar", que permite salvar os dados em formato ASCII.

\section{Experimentos e Resultados}

Um experimento foi desenvolvido com o objetivo de avaliar a integração da rede de sensores de temperatura e o software para o controle da estação total. Neste, os cinco sensores que foram instalados no entorno e em áreas de monitoramento geodésico da barragem Salto Caxias, no estado do Paraná. Tal barragem foi escolhida em razão desta contar com uma infraestrutura adequada para o monitoramento geodésico, viabilizando o teste do sistema proposto e a integração de uma rede de sensores de temperatura durante a 
medição de pontos de monitoramento. A rede de monitoramento geodésico desta barragem é externa (Figura 8), composta por pilares de centragem forçada (EGE06, EGE04, EGE05, EGE03, EGE01) e um ponto engastado na estrutura também de centragem forçada (C14). Na mesma Figura 8 é possível identificar os sensores de temperatura dispostos na barragem (S1, S2, S3, S4, S5) e na posição do módulo coordenador (CC).

Figura 8 - Rede de monitoramento geodésico

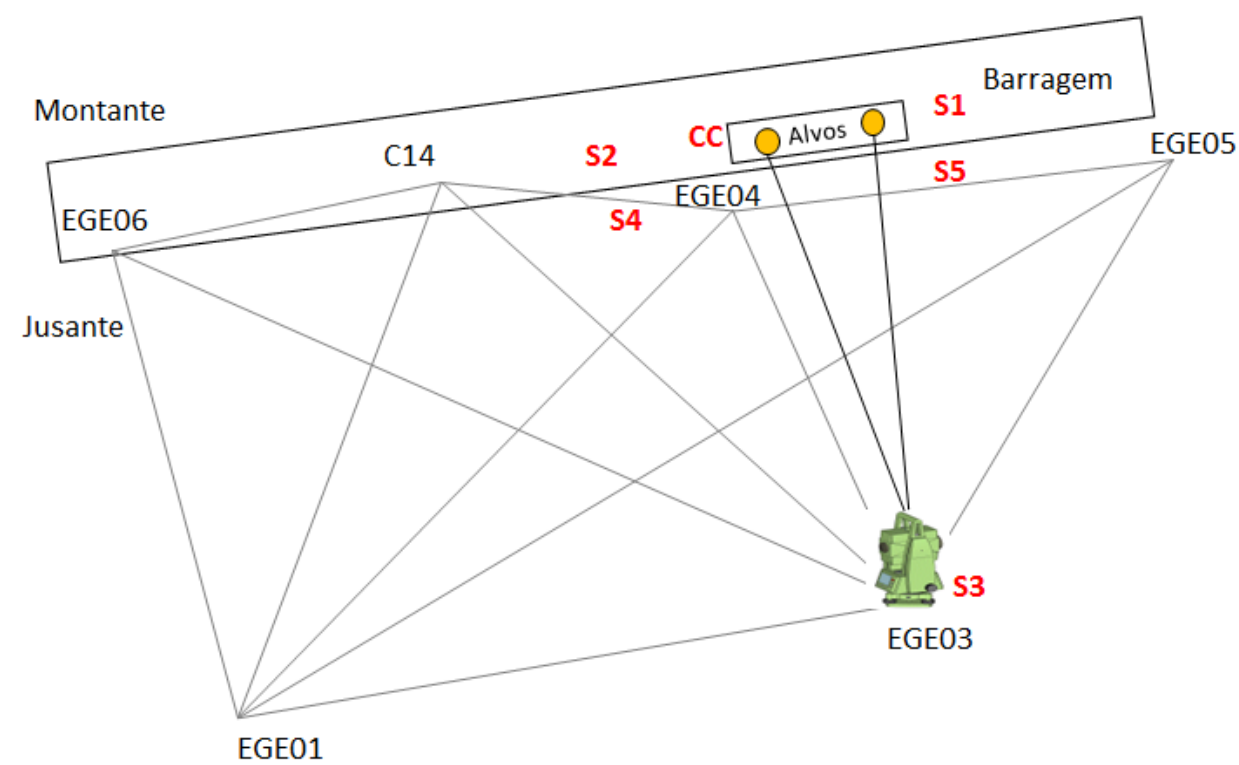

Fonte: Elaborada pelos autores.

Neste teste, a estação total foi instalada no pilar EGE03 e as medições foram feitas nos alvos fixos à jusante da barragem, com uma distância aproximada de $358 \mathrm{~m}$ (ver Figura 8). Os sensores de temperatura foram colocados junto ao instrumento de medição (S3), nas posições próximos à região onde se encontram os pontos objeto que são monitorados (S1 e S2) e na crista da barragem (S4 e S5) para avaliar a influência da massa de água durante o monitoramento. $\mathrm{O}$ módulo coordenador (CC) também se encontra próximo aos pontos objeto. A avaliação do sistema, por sua vez, foi feita a partir da medição de distâncias a um alvo à jusante e leitura dos sensores de temperatura. 
Os resultados apresentados no gráfico da Figura 9 mostram a variação de temperatura durante a medição de pontos de monitoramento geodésico.

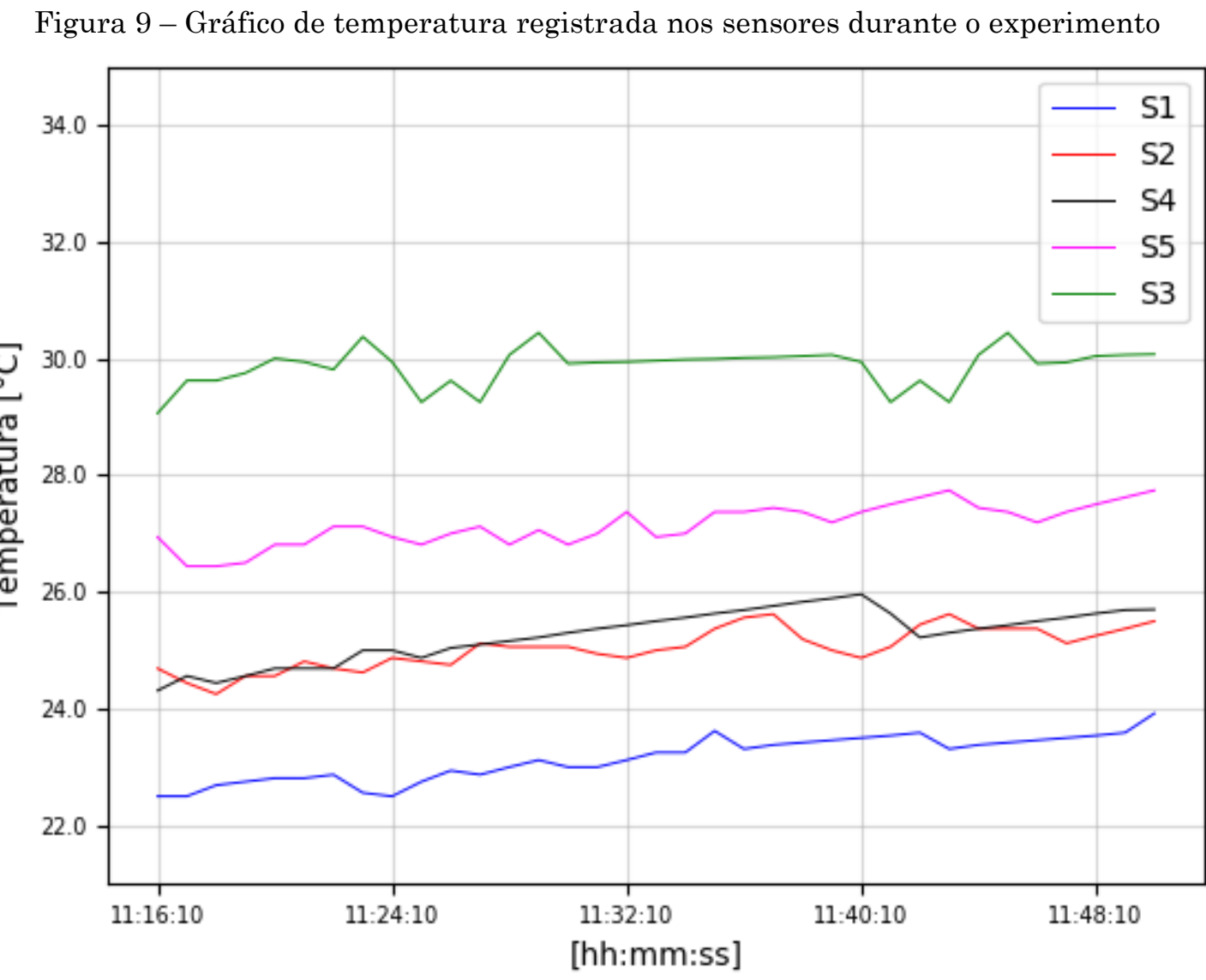

Fonte: Carvajal (2018).

Estes valores indicam variações na temperatura registrada pelos sensores com diferenças de temperatura de até $5^{\circ} \mathrm{C}$. Na região da crista da barragem foram registradas as menores temperaturas, influenciadas principalmente pela massa de água do lado à montante. Para o caso dos sensores instalados na jusante, estes apresentam um aumento de temperatura em comparação aos sensores instalados na crista da barragem em consequência do calor dissipado pela mesma. No local onde a estação total está instalada os valores são maiores devido ao ponto estar localizado em uma região de vale, onde a radiação de calor causa gradientes verticais irregulares de temperatura em decorrência das diferentes capacidades de 
absorção e emissão de calor. No caso específico do pilar EGE03, este localizase numa região onde os elementos em seu entorno encontram-se mais elevados, gerando um encapsulamento de temperatura em sua posição (CARVAJAL, 2018).

Com os resultados obtidos, foram calculadas as distâncias corrigidas dos efeitos atmosféricos a partir de três maneiras diferentes:

a) Temperatura medida no sensor instalado junto à estação total, representando o procedimento padrão executado em trabalhos de mensuração $(t 1)$;

b) Temperatura média dos cinco sensores ( $t 2)$; e

c) Temperatura média dos dois sensores mais próximos dos extremos da linha visada $(t 3)$.

Utilizou-se a estação total modelo TS15 da Leica, e a modelagem de correção de parâmetros atmosféricos é apresentada na Equação 1 (LEICA, 2015).

$$
\Delta D_{1}=286,34-\left[\frac{0,29525 \cdot p}{(1+\alpha \cdot \mathrm{t})}-\frac{4,126 \cdot 10^{-4}}{(1+\alpha \cdot t)} \cdot 10^{x}\right]
$$

onde:

$$
\begin{aligned}
& \Delta \mathrm{D} 1: \text { Correção atmosférica }[\mathrm{ppm}] \\
& \text { p: pressão }[\mathrm{mbar}] \\
& \text { t: temperatura }\left[{ }^{\circ} \mathrm{C}\right] \\
& \text { h: umidade relativa }[\%] \\
& \mathrm{a}=1 / 273 \\
& \mathrm{x}=[(7,5 \cdot \mathrm{t}) /(273,15+\mathrm{t})]+0,7857
\end{aligned}
$$

A partir das temperaturas $t 1, t 2$ e $t 3$, foram obtidas as correções $c 1, c 2$, c3 e, em seguida, aplicadas à distância eletrônica (sem correção e inclinada) entre o pilar de monitoramento EGE03 e o ponto F01 à jusante da barragem, apresentados na Figura 10. 
Figura 10 - Distância entre o pilar de monitoramento e o alvo escolhido para o teste

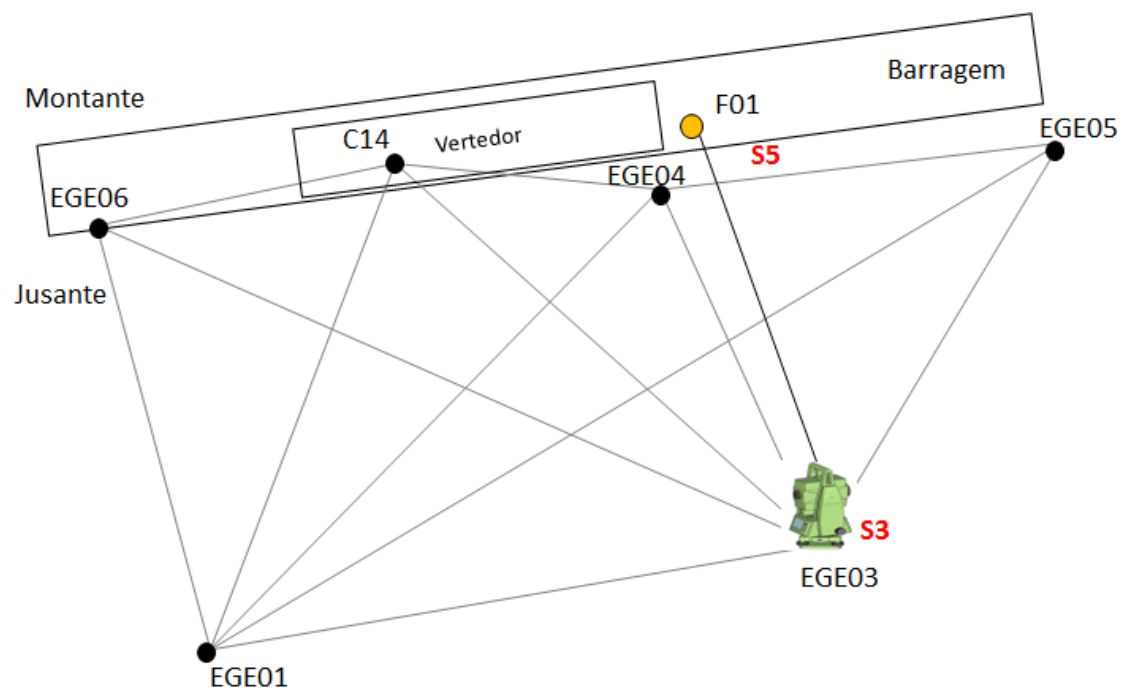

Fonte: Elaborada pelos autores.

Por fim, a correção é feita a partir da equação 2 (RÜEGER, 1990):

$$
c_{n}=\Delta D_{1} \cdot D c
$$

onde:
$\Delta \mathrm{D}_{1}$ : Correção atmosférica $[\mathrm{ppm}]$
Dc: Distância fornecida pelo instrumento [m] $c_{n:} n$ correções de velocidade

A distância inclinada neste teste foi de 358,6846 m e as correções obtidas são apresentadas no gráfico apresentado na Figura 11. 
Figura 11 - Correções das distâncias obtidas a partir de $c_{1}, c_{2}, c_{3}$

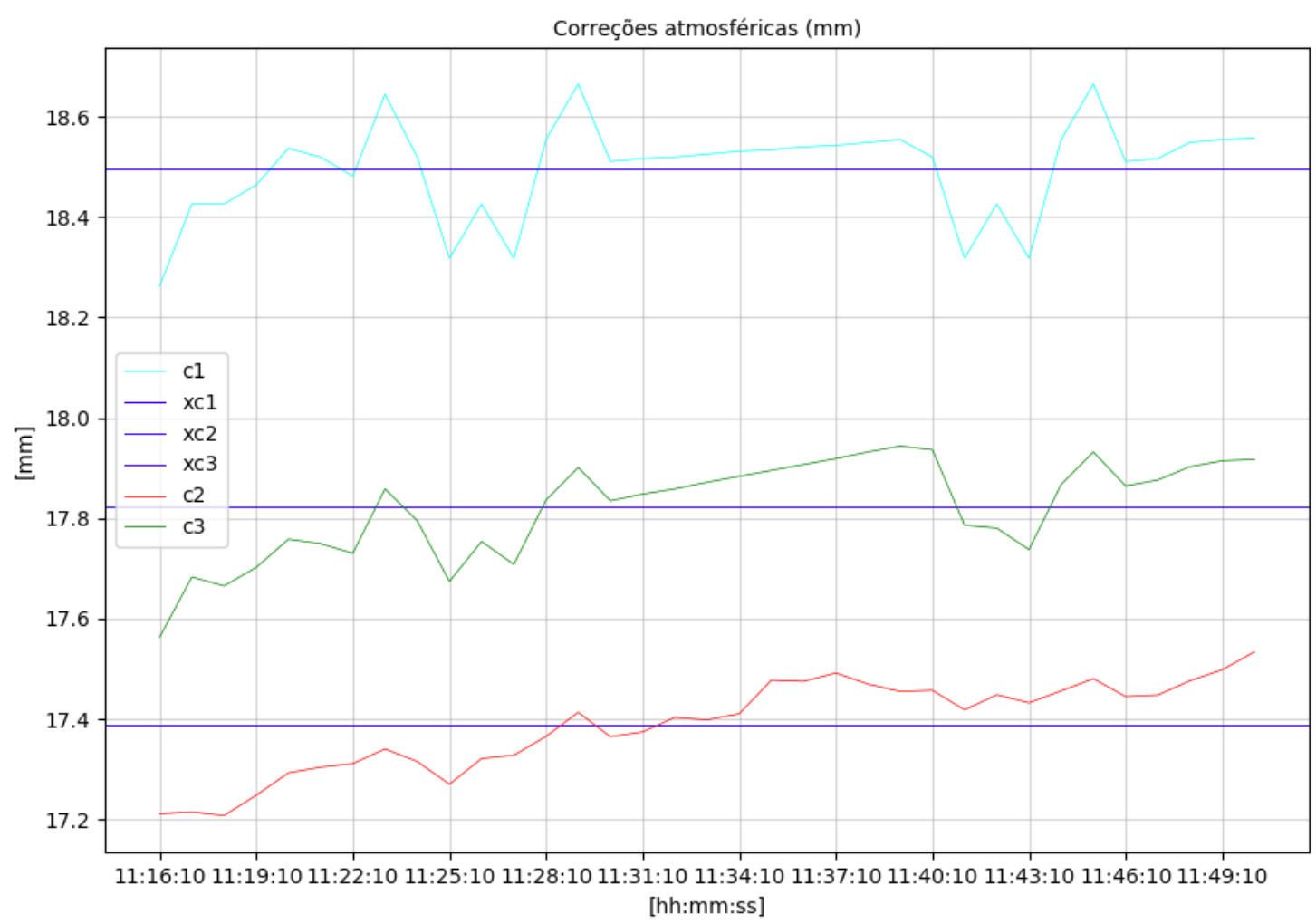

Fonte: Elaborada pelos autores.

É possível observar que conforme a opção utilizada para o cálculo da temperatura a ser aplicada na correção do índice de refração, pode-se chegar a uma diferença média de $1 \mathrm{~mm}$ e máximas de 1,7 $\mathrm{mm}$. Estas diferenças geradas pelas condições ambientais, que envolvem o monitoramento geodésico da estrutura, podem afetar a correta determinação dos descolamentos dos pontos monitorados e as análises da deformação da estrutura.

Para a distância medida neste experimento $(358,6846 \mathrm{~m})$, as variações médias $(1 \mathrm{~mm})$ correspondem à $0,28 \mathrm{ppm}$. Porém, os valores de temperatura usados para as correções $c 2$ e $c 3$ são mais representativos da região, devido, principalmente, à possibilidade de diferentes distribuições dos sensores. $\mathrm{O}$ uso dos valores médios $x c 1, x c 2$ e $x c 3$ para as correções fundamenta-se no fato destes representarem o estado da temperatura durante o teste. Porém, os valores $c 2$ e $c 3$ apresentaram um aumento na temperatura, o qual não é 
evidenciado no valor $c 1$. Dessa forma os valores médios $x c 2$ e $x c 3$ são obtidos em função deste aumento, sendo considerados mais representativos.

\section{Conclusões}

Para que um processo de monitoramento possa garantir a qualidade das observações geradas, faz-se necessário um rigoroso controle de todas as operações e observações realizadas, o que impulsiona a busca constante por métodos que possam contribuir com este objetivo. Neste ponto, a automação pode ajudar na diminuição do tempo de resposta a um evento, minimizar possíveis erros de observação, padronizar operações e permitir que sejam acrescentados dados auxiliares às observações básicas em monitoramento realizado por técnicas terrestres tradicionais, envolvendo a medida de distâncias e, consequentemente, o efeito dos parâmetros ambientais sobre a mesma deve ser considerado, destacadamente a questão da temperatura.

Nesse sentido, o desenvolvimento do software abordado neste trabalho permitiu o controle de uma estação total em tempo real, mediante o envio e recepção de códigos de controle, padronizando-se o formato dos códigos para tornar mais eficiente a comunicação entre o instrumento de medida e o software desenvolvido. Ademais, foi programada uma interface gráfica para a coleta e gerenciamento dos dados observados, com vistas a torná-la mais intuitiva ao usuário. Além do controle das funcionalidades da estação total, foi possível integrar e sincronizar valores observados de temperatura em tempo real fornecidos por uma rede de sensores sem fio em simultâneo às medições eletrônicas de distância.

A integração da rede de sensores de temperatura permitiu considerar não somente a temperatura pontual junto ao pilar, onde está instalado o equipamento, mas na área que abrange a medida da distância e, assim, estimar os parâmetros de diferentes formas para realizar a primeira correção de velocidade. 
Os resultados apresentados no gráfico da Figura 9 mostram a variação de temperatura durante a medição de pontos de monitoramento geodésico de uma barragem. Os valores apresentaram diferenças que alcançaram $5^{\circ} \mathrm{C}$, além da variação de temperatura em cada ponto ter sido diferente ao longo do experimento. A correção na distância, por sua vez, alcançou uma diferença máxima de $1,7 \mathrm{~mm}$ para um intervalo de aproximadamente 30 minutos. Foram utilizadas três formas de calcular a temperatura. A primeira, mais tradicional, foi o uso da temperatura medida junto ao pilar onde a estação está instalada, na qual o valor utilizado apresentou uma variação de cerca de $5^{\circ} \mathrm{C}$ em relação à posição onde estavam os pontos monitorados. No segundo caso foi utilizada a abordagem apresentada por Rüeger (1990), na qual estimou-se como um melhor valor para a temperatura a média dos valores de temperatura nos extremos da linha. Uma terceira situação foi realizada através da média da temperatura, levando em consideração todos os sensores da rede. Conforme analisado, esta diferença máxima $(1,7 \mathrm{~mm})$ pode representar mais de 0,28 ppm da distância medida, valor significativo para o monitoramento de estruturas (US ARMY CORPS OF ENGINEERS 2018).

Considerando que a variação de $1^{\circ} \mathrm{C}$ proposta por Rüeger (1990) gera um 1 ppm de incerteza no índice de refração,e que esta é calculada para uma medição pontual, a correção obtida a partir do sistema de aquisição de temperatura tornou possível obter resultados mais representativos, por meio das distribuições espaciais dos sensores e das modelagens da temperatura.

A utilização do software e da rede mostrou-se eficaz no sentido de permitir a escolha do método de correção da temperatura diretamente em campo e em tempo real. Além disso, o desenvolvimento do software e a integração da rede de sensores de temperatura permitirão pesquisar outras técnicas ou métodos para obter um valor de temperatura mais representativo na área de estudos. 


\section{Agradecimentos}

Os autores agradecem a Copel - Companhia Paranaense de Energia por permitir a execução dos nos testes na usina de Salto Caxias. Os autores também agradecem a CAPES pela bolsa de estudos (número de processo 40001016002P6).

\section{Contribuição dos autores}

O artigo apresentado foi realizado no período do mestrado acadêmico do autor principal, Felipe Carvajal Rodriguez sob orientação do segundo autor Luis Augusto Koenig Veiga, quem realizou as correções do trabalho e forneceu ajuda no desenvolvimento dos experimentos.

\section{Referências}

ANGUS-LEPPAN, P. V; BRUNNER, F. K. Atmospheric temperature models for short-range E.D.M. The Canadian Surveyor, v. 34 (2), 1980, pp. 153-165.

ANGUS-LEPPAN. Adjustment of trilateration using length ratios, Survey Review, vol 21, 1972, pp.355-368.

ARTESE S.; PERRELLI, M. Monitoring a landslide with high accuracy by total station: a DTM-based model to correct for the atmospheric effects. Geosciences. vol. 8(2), 2018.

BRUNNER, F.K. Geodetic Refraction. $1^{a}$ ed. Berlin Heidelberg. Springer, 1984. $216 \mathrm{p}$

BRUNNER, F. K.; FRASER, C. S. An atmospheric turbulent transfer model for EDM reduction. International Symposium on Electromagnetic Distance Measurement and the Influence of Atmospheric Refraction, $1977 \mathrm{pp}$. 304-317.

CARVAJAL R. F. A. Automação da correção da primeira velocidade em medidas eletrônicas de distância com base em rede de observação multi-estações. Dissertação de mestrado. Universidade federal do Paraná. Programa Pós-Graduação em Ciências Geodésicas, Curitiba, 2018. 157p. 
FRASER, C. S. A simple atmospheric model for electro-optical EDM reduction. Australian Surveyor, vol. 30, 1981 pp. 352-362.

KENNIE, T. J. M.; PETRIE, G. Engineering Surveying Technology. $1^{\mathrm{a}}$ ed. Oxon: Taylor \& Francis, 1993. 508p.

LEICA GEOSYSTEMS. GSI ONLINE for Leica TPS. Advanced User Guide. Leica Geosystems AG, Switzerland, 1999.38p

LEICA GEOSYSTEMS. GeoCOM Reference Manual. Manual de referência. Leica Geosystems AG, Switzerland, 2009.131p

LEICA GEOSYSTEMS. Leica TS15 user manual. Leica Geosystems AG, Switzerland, 2015.110p

NMEA 0183: Standard for Interfacing Marine Electronic Devices. National Marine Electronics Association, 2002.

OGUNDARE, J. O. Precision Surveying: The Principles and Geomatics Practice, $1^{\mathrm{a}}$ ed. Hoboken: John Wiley \& Sons, 2015. 648p.

ROBERTSON, K. D. The use of atmospheric models with trilateration. Survey Review, vol. 24, 1977 pp. 179-188.

RÜEGER, J. M. Electronic Distance Measurement: An Introduction. $4^{\mathrm{a}}$ ed. Sydney: Springer, 1990.

SOLARIĆ N.; BARKOVIĆ, Đ.; ZRINJSKI, M. Automation of the Measurement of Atmospheric Parameters in Precise Distance Measurement. Geodetski List, vol. 66,2012 , pp.165-186.

TORGE, W. Geodesy. $4^{\mathrm{a}}$ ed. Berlin, New York: Degruyter, 2012.444p.

US ARMY CORPS OF ENGINEERS. Structural Deformation Surveying. Engineering and design, 2018, Em1110-2-1009. Pp. 365.

VEIGA, L. A. K. Sistema para Mapeamento Automatizado em campo: conceitos, metodologia e implementação de um protótipo. Tese de doutoramento. Universidade de são Paulo Programa Pós-Graduação em Engenharia de Transportes Universidade de São Paulo. São Paulo, 2000. 